\title{
Dibromomaleimide as a facile, versatile linker for delivery applications
}

\author{
"The ease and versatility of dibromomaleimide chemistry has \\ allowed the reversible attachment of biomolecules in a wide \\ variety of applications such as polymer synthesis, cancer, and \\ gene delivery."
}

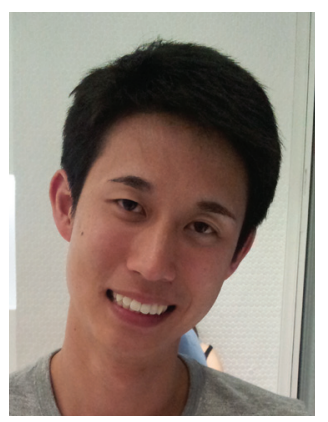

James-Kevin Y Tan Department of Bioengineering \& Molecular Engineering \& Sciences Institute, University of Washington, William H Foege Building, Box 355061, 3720 15th Ave NE, Seattle, WA 98195, USA

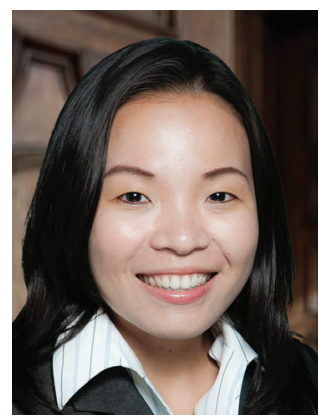

Joan G Schellinger

Author for correspondence: Department of Chemistry \& Biochemistry, University of San Diego, 5998 Alcala Park, San Diego, CA 92110, USA

jschellinger@sandiego.edu Caddick $[1,2]$, a wide variety of thiol molecules have been substituted onto DBM such as peptides [2-4], proteins [2,5,6] and polymers $[7,8]$. DBM's utility has been further expanded as a fluorescent tag; when small alkyl chains are substituted to the DBM molecule, the resulting conjugate exhibits bright fluorescence in a range of solvents $[9,10]$. DBM also features a unique ability to exchange

\section{Polymer synthesis applications}

A wide variety of polymer architectures such as linear, branched, star, and dendrimer shapes are being developed with their own distinct routes of efficient synthesis [12]. As a small linker with three points of attachment, DBM has the ability to reversibly connect polymers together and provide site-specific functionalization by relatively simple chemical reactions. Cui et al. utilized DBM as the central connector of three polymer chains [7]. The DBM bromines were substituted with either poly(ethylene oxide) (PEG), poly $(N$-(2-acryloyloxyethyl) pyrrolidone) or $\operatorname{poly}(N, N$-dimethyl acrylamide) while the imide of DBM had a bromobutylene that was changed into an azido unit for the attachment of another PEG polymer by copper-catalyzed azide-alkyne cycloaddition. In another example, polymers were synthesized

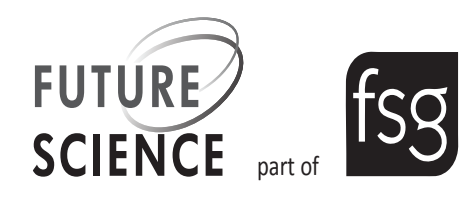


in the presence of unprotected DBM. Robin et al. synthesized a DBM and reversible addition chain-transfer macroinitiator and polymerized butyl acrylate, methyl methacrylate, and triethylene glycol methyl ether acrylate with controlled size and polydispersity [13]. While materials design and synthesis continue to create advanced architectures with multiple functionalities by complex chemistries, DBM can provide simpler methods of polymer attachment, chemical functionalization, and degradation to create structures of great complexity for a variety of delivery applications.

\section{Imaging applications}

In all delivery applications, the ability to track and monitor the cargo is important in understanding the delivery location, mechanism and distribution. Since DBM is a versatile, small molecule linker that fluoresces upon conjugation with a variety of thiolcontaining ligands $[10,14,15]$, it can be used in imaging and vehicle tracking applications. By substituting DBM with different thiols, Robin et al. prepared different dithiomaleimide (DTM) small molecules that have bright emissive fluorescence properties at about $500 \mathrm{~nm}$ [15]. In addition, they have shown that these DTM fluorophores can be readily incorporated to polymers and proteins. Similarly, they have developed DTM-based fluorescent polymers that can selfassemble into nanoparticles and be used as self-reporting probes for delivery applications. Most recently, Wang et al. prepared a DTM-based camptothecin nanoparticle used as an anticancer drug delivery system [16]. This nanoparticle showed high drug loading and was FRET responsive by taking advantage of the intracellular glutathione concentration. The inherent fluorescence of DBM upon conjugation allows for the design and synthesis of a new class of materials for the imaging and tracking of particles, including small molecule drug release. These materials are capable of facile assembly and disassembly, a desirable feature for monitoring therapeutic agent delivery.

\section{Cancer treatment applications}

Anticancer drugs are known to cause severe indiscriminate side effects when administered systemically. Thus, cancer treatments focus on delivering drugs specifically to cancer cells by incorporating cancerhoming moieties, such as small molecules, peptides and proteins into their delivery systems. One construct of growing interest is the antibody-drug conjugate, which involves the specific attachment of a cytotoxic drug to an antibody (Ab). With the appropriate conjugation technique, these 'magic bullets' offer distinct advantages of homogeneity, high yield, and long stability without undesirable toxic side effects [17]. There- fore, the choice of conjugation method in antibodydrug conjugate synthesis is of crucial importance to its cancer treatment application.

"DBM is an adaptable linker that can be ... used to bridge peptides, fluorophores, antibodies, and polymers."

DBM offers a facile and promising conjugation strategy that allows the use of the Ab's native disulfide bonds in the attachment and delivery of chemotherapeutic drugs. For example, the Fab fragment and the functional bridging disulfide interchain of trastuzumab (Herceptin ${ }^{\circledR}$ ) was utilized and conjugated to an alkynefunctionalized DBM that can be readily reacted with azide-containing drugs such as porphyrins and doxorubicin $[18,19]$. The chemical reactions were mild enough to retain $\mathrm{Ab}$ binding, an important attribute for $\mathrm{Ab}$-based cancer therapy. One distinguishing advantage of the use of DBM chemistry is the abolishment of the need for $\mathrm{Ab}$ functionalization by protein engineering. Utilizing the native Ab disulfide bonds removes any additional complicated steps for Ab production, thus making it simpler and more economical. While some issues may arise, such as formation of a half $\mathrm{Ab}$ due to intrachain disulfide bridging [19] and changes in protein function upon insertion of DBM, optimizing the reaction conditions and utilizing the appropriate DBM linker may potentially address these challenges. Nevertheless, this technology presents a novel way to conjugate a native $\mathrm{Ab}$ to a drug while keeping the Ab's structural features intact. In addition, the degradation of DBM in the reducing condition of the cell cytoplasm can allow for the release of the anticancer drug for a more potent effect, a feat not possible for nonlabile linkers.

\section{Nonviral gene delivery applications}

In nonviral gene delivery, typically cationic polymers are complexed with negatively charged nucleic acids to form a gene transfer vehicle. Polyethylenimine (PEI) is one of the most common and effective cationic polymers utilized for gene delivery; however, its high density of positive charge and nondegradable nature limit its application in vivo [20]. To surmount this shortcoming, degradable PEI and other cationic polymers have been prepared to decrease cytotoxicity in vitro by dissociating into smaller, less toxic pieces.

The chemical versatility of DBM allows for the development of an ideal nonviral vector capable of addressing the barriers of gene delivery such as high toxicity and the release of nucleic acids after internalization into cells. The ability of DTM conjugates for thiol exchange by intracellular glutathione allows for these nucleic acid-polymer complexes to be cleaved in vivo; thus allowing for the dissociation of a large density of 
cationic charge in the cell cytoplasm and nucleic acid release. Furthermore, DBM allows for the development of multifunctional polymers by further functionalization at the $N$-maleimide moiety with small molecules, such as fluorophores, and macromolecules, such as peptide and proteins. To showcase these features, Tan et al. synthesized a panel of cleavable cationic polymers incorporating DBM as a reversible linker to bridge statistical copolymers of (2-dimethyl-amino)ethyl methacrylate and oligo(ethylene glycol)methyl ether methacrylate [8]. These polymers exhibited better cell viability than the nonreducible analogs, while maintaining gene transfer capabilities. In addition, a fluorophore was incorporated and cellular uptake was monitored by flow cytometry. The versatility and degradation of DBM allow for an apropos application in gene delivery.

Overall, DBM is an adaptable linker that can be applied to a wide variety of molecules and applications.

\section{References}

1 Tedaldi LM, Smith MEB, Nathani RI, Baker JR. Bromomaleimides: new reagents for the selective and reversible modification of cysteine. Chem. Comm. (43), 6583-6585 (2009).

2 Smith MEB, Schumacher FF, Ryan CP et al. Protein modification, bioconjugation, and disulfide bridging using bromomaleimides. J. Am. Chem. Soc. 132(6), 1960-1965 (2010).

3 Ryan CP, Smith MEB, Schumacher FF et al. Tunable reagents for multi-functional bioconjugation: reversible or permanent chemical modification of proteins and peptides by control of maleimide hydrolysis. Chem. Comm. 47(19), 5452-5454 (2011).

4 Schumacher FF, Nobles M, Ryan CP et al. In situ maleimide bridging of disulfides and a new approach to protein pegylation. Bioconjug. Chem. 22(2), 132-136 (2011).

5 Jones MW, Strickland RA, Schumacher FF et al. Polymeric dibromomaleimides as extremely efficient disulfide bridging bioconjugation and pegylation agents. J. Am. Chem. Soc. 134(3), 1847-1852 (2012).

6 Jones MW, Strickland RA, Schumacher FF et al. Highly efficient disulfide bridging polymers for bioconjugates from radical-compatible dithiophenol maleimides. Chem. Comm. 48(34), 4064-4066 (2012).

7 Cui Y, Yan Y, Chen Y, Wang Z. Dibromomaleimide derivative as an efficient polymer coupling agent for building topological polymers. Macromol. Chem. Physic. 214(4), 470-477 (2013).

8 Tan J-KY, Choi JL, Wei H, Schellinger JG, Pun SH. Reducible, dibromomaleimide-linked polymers for gene delivery. Biomater. Sci. 3(1), 112-120 (2015).

9 Onimura K, Matsushima M, Nakamura M, Tominaga T, Yamabuki K, Oishi T. Synthesis and fluorescent properties of model compounds for conjugated polymer containing maleimide units at the main chain. J. Polym. Sci. Pol. Chem. 49(16), 3550-3558 (2011).
It has already been used to bridge peptides, fluorophores, antibodies, and polymers. Meanwhile, its degradation in reducing conditions affords an advantage in imaging, cancer, and gene delivery applications that is not obtainable with nonreducing linkers. DBM's facile versatility has already allowed many applications, with a great promise of more to come.

\section{Financial \& competing interests disclosure}

The authors have no relevant affiliations or financial involvement with any organization or entity with a financial interest in or financial conflict with the subject matter or materials discussed in the manuscript. This includes employment, consultancies, honoraria, stock ownership or options, expert testimony, grants or patents received or pending, or royalties.

No writing assistance was utilized in the production of this manuscript.

10 Robin MP, Wilson P, Mabire AB et al. Conjugationinduced fluorescent labeling of proteins and polymers using dithiomaleimides. J. Am. Chem. Soc. 135(8), 2875-2878 (2013).

11 Moody P, Smith MEB, Ryan CP et al. Bromomaleimidelinked bioconjugates are cleavable in mammalian cells. Chem. Biochem. 13(1), 39-41 (2012).

12 Qiu L, Bae Y. Polymer architecture and drug delivery. Pharm. Res. 23(1), 1-30 (2006).

13 Robin MP, Jones MW, Haddleton DM, O'Reilly RK. Dibromomaleimide end functional polymers by raft polymerization without the need of protecting groups. ACS Macro Lett. 1(1), 222-226 (2012).

14 Robin MP, O’Reilly RK. Fluorescent and chemicofluorescent responsive polymers from dithiomaleimide and dibromomaleimide functional monomers. Chem. Sci. 5(7), 2717-2723 (2014).

15 Robin MP, Mabire AB, Damborsky JC et al. New functional handle for use as a self-reporting contrast and delivery agent in nanomedicine. J. Am. Chem. Soc. 135(25), 9518-9524 (2013).

16 Wang $\mathrm{H}, \mathrm{Xu} \mathrm{M}$, Xiong M, Cheng J. Reduction-responsive dithiomaleimide-based nanomedicine with high drug loading and fret-indicated drug release. Chem. Comm. 51(23), 4807-4810 (2015).

17 Kline T, Steiner A, Penta K, Sato A, Hallam T, Yin G. Methods to make homogenous antibody drug conjugates. Pharm. Res. 1-14 (2014).

18 Bryden F, Maruani A, Savoie H et al. Regioselective and stoichiometrically controlled conjugation of photodynamic sensitizers to a HER 2 targeting antibody fragment. Bioconjug. Chem. 25(3), 611-617 (2014).

19 Schumacher FF, Nunes JP, Maruani A et al. Next generation maleimides enable the controlled assembly of antibody-drug conjugates via native disulfide bond bridging. Org. Biomol. Chem. 12(37), 7261-7269 (2014).

20 Fischer D, Li Y, Ahlemeyer B, Krieglstein J, Kissel T. In vitro cytotoxicity testing of polycations: influence of polymer structure on cell viability and hemolysis. Biomaterials 24(7), 1121-1131 (2003). 